\title{
AV block after flutter ablations?
}

\author{
A. W. G. J. Oomen • L. R. C. Dekker • A. Meijer
}

Published online: 8 May 2014

(C) The Author(s) 2014. This article is published with open access at Springerlink.com

\section{Rhythm Puzzle-Question}

A 68-year-old, otherwise healthy male, presented at the emergency department with dizziness and near-syncope 4 days after a redo catheter ablation procedure for atypical right atrial flutter.

In 1980 he underwent surgical closure of an atrial septal defect. One year prior to the current problem, a catheter ablation procedure in the right atrium was performed because of an atypical isthmus-dependent atrial flutter. During this procedure the cavotricuspid isthmus was blocked. Four days before presentation at the emergency department, a redo procedure was performed because of another postincisional atrial flutter. This time the flutter circuit was located in the septum and was successfully ablated. The earlier ablation lines were well recognisable and still formed block.

Physical examination and laboratory tests did not demonstrate any abnormalities.

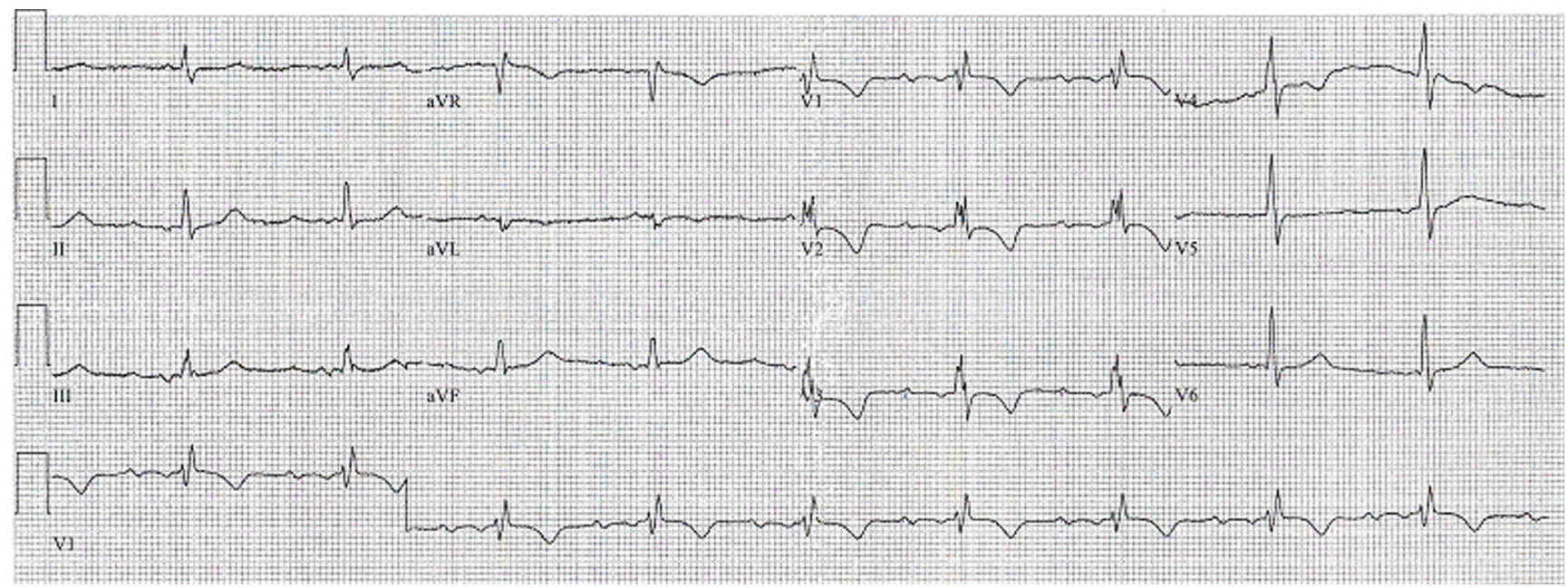

Fig. 1 Electrocardiogram on admission

A. W. G. J. Oomen $(\bowtie)$

Department of Cardiology, Catharina Ziekenhuis, Michelangelolaan

2, Eindhoven 5623 EJ, the Netherlands

e-mail: adoomenjr@gmail.com

L. R. C. Dekker • A. Meijer

Catharina Hospital, Michelangelolaan, Eindhoven 5623 EJ,

the Netherlands 


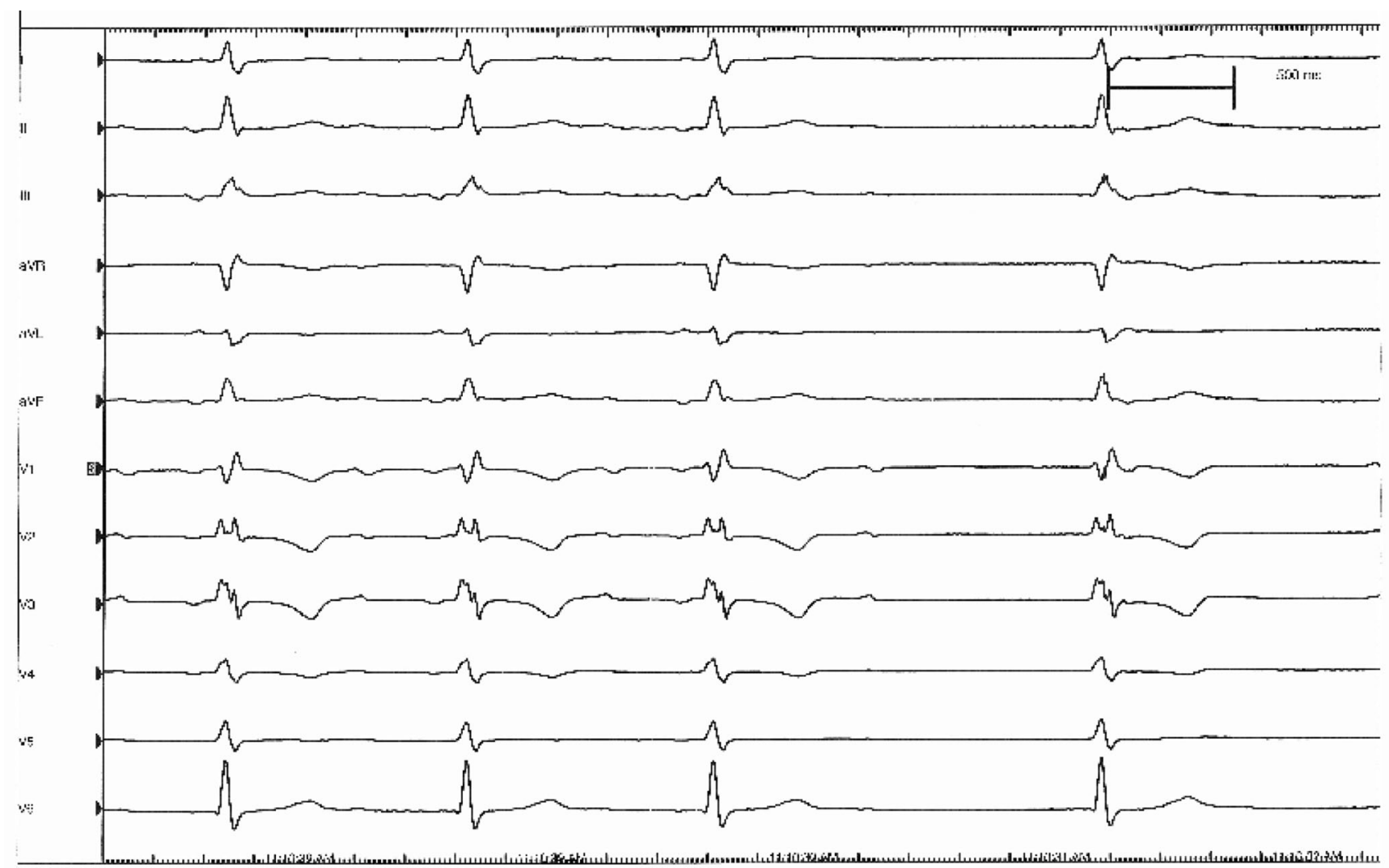

Fig. 2 Rhythm recording in cathlab with paper speed $50 \mathrm{~mm} / \mathrm{s}$

A 12-lead electrocardiogram and rhythm recording after his re-admission for near-syncope are shown in Fig. 1 and 2 respectively. What is your diagnosis and what is the mechanism?
Open Access This article is distributed under the terms of the Creative Commons Attribution License which permits any use, distribution, and reproduction in any medium, provided the original author(s) and the source are credited. 